\title{
О ДИНАМИКЕ АВТОНОМНЫХ СИСТЕМ, КАНОНИЗИРУЕМЫХ ПРЕОБРАЗОВАНИЕМ ВРЕМЕНИ
}

I. KEIS. AJA JARGI KANOONILISELE KUJULE TEISENDATUD AUTONOOMSETE SUSTEEMIDE DUNAAMIKAST

I. KEIS. ON THE DYNAMICS OF THE AUTONOMOUS SYSTEMS TIME-REDUCIBLE TO THE CANONICAL FORM

\author{
(Представил Н. Алумяэ)
}

В работе, продолжающей исследования $\left[{ }^{1,2}\right]$, рассмотрена задача определения вида управлений и свойств непотенциальной системы

$$
\begin{gathered}
q=\partial H / \partial p, \quad p=-\partial H / \partial q+u(q, p) \\
\left(\Delta_{p} H \equiv\left|\partial^{2} H / \partial p_{i} \partial p_{j}\right| \neq 0, u \in C_{1}(q, p), \operatorname{dim} q, p=\overline{1, n}\right),
\end{gathered}
$$

приводимой дифференциальным преобразованием $d \tau / d t=\lambda$ к канонической форме

$$
\begin{gathered}
q^{\prime}=\partial G / \partial P, \quad P^{\prime}=-\partial G / \partial q \\
\left(d f / d t=f=\lambda f^{\prime}, f^{\prime}=d f / d \tau, q=\lambda q^{\prime}, G=G(q, P)\right)
\end{gathered}
$$

$$
\tilde{\lambda}+q^{\prime} \cdot \nabla_{q^{\prime}} \tilde{\lambda} \neq 0, \quad \tilde{\lambda}\left(q, q^{\prime}\right)=\lambda(q, P)=\lambda \in C_{1}(q, P) \quad\left(\Delta_{P} G \neq 0\right),
$$

$\left\{\lambda=0 / q^{\prime}=0, P^{\prime}=0\right\} \equiv Q \notin I-$ инвариантное множество (2).

Рассмотрим сперва позиционный $\left[{ }^{1}\right]$ случай $\lambda=\lambda(q)$. Обозначим

$$
\begin{gathered}
L(q, q)=q \cdot p-H, \quad \tilde{L}\left(q, q^{\prime}\right) \equiv L\left(q, \lambda q^{\prime}\right), \\
K\left(q, q^{\prime}\right)=q^{\prime} \cdot P-G, \quad R\left(q, q^{\prime}\right)=K-\tilde{L}, \\
r(q, P)=\nabla_{q^{\prime}} R, \quad N(q, P) \equiv q^{\prime} \cdot r-R=G-H, \quad \lambda p=P-r, \\
\lambda \cdot(q, p)=\lambda(q, P), \\
\left.p=\partial L /\left.\partial q \cdot\right|_{q^{*}=g \equiv \psi(q, P),} ^{n}{ }^{n} a_{s=1} b_{s}\right),
\end{gathered}
$$

$$
q=g(q, P) \equiv \lambda \partial G / \partial P, \quad q^{\prime}=\lambda_{*}^{-1} \partial H / \partial p \equiv h(q, p)\left(r=\left(r_{i}\right)^{*}, i, s=\overline{1, n}\right),
$$

где $H, G-$ заданные нормальные функции $\left(\Delta_{p} H \neq 0, \Delta_{P} G \neq 0\right)$. Из (1) - (3) находим для них искомую структуру вектор-функции регулятора 


$$
\begin{gathered}
u=v_{0}(q, p)=(q \cdot p) \frac{\partial \mu}{\partial q}-\left(q \cdot \frac{\partial \mu}{\partial q}\right) p-\left(\frac{\partial r}{\partial P} \cdot P^{\prime}\right)-\frac{\partial N}{\partial q} \\
\left(\mu \equiv \ln |\lambda|, f^{\prime}=\frac{d f}{d \tau}\right) .
\end{gathered}
$$

В силу (4) и тождеств $G^{\prime} \equiv 0, q_{s}^{\prime} \partial r_{s} / \partial P_{j} \equiv \partial N / \partial P_{j}(j=\overline{1, n)}$ его мощность $W\left(v_{0}\right) \equiv q \cdot v_{0}=-\lambda N^{\prime}$. Следовательно, для регулятора приводимой системы $W\left(v_{0}\right) \neq 0$ при $\lambda=\lambda(q), N^{\prime} \neq 0$. Подслучаем данного будет вариант подобных по $p, P$ функций $H, G(G(q, P) \equiv H(q, \psi(q, P)))$. В нем обобщены на $n>2$ результаты, полученные для ряда неголономных систем $\left[\left[^{1}\right]\right.$. Можно показать, что в этом варианте подобие $H, G$ эквивалентно равенствам $P=\lambda p\left(P \cdot q^{\prime}=p \cdot q^{*}\right)$ и подобию $L, K$ по $q^{*}, q^{\prime}\left(K\left(q, q^{\prime}\right) \equiv L\left[q, g\left(q, \partial K / \partial q^{\prime}\right)\right], r \equiv 0, R \equiv N \equiv 0\right)$. Тогда из $(2)$ -(4) находим приводящий регулятор системы (1)

$\tilde{v}_{0}=\Gamma q, \quad \Gamma=\left\|\gamma_{i j}\right\|=-\Gamma^{*}, \quad \gamma_{i j}=p_{j} \partial \mu / \partial q_{i}-p_{i} \partial \mu / \partial q_{j} \quad(i, j=\overrightarrow{1, n})$.

При этом $W \equiv 0, H$ - инвариант системы (1), $H, \tilde{v}_{0 i}-$ полиномы одной степени по $p(\mu=\ln |\lambda| \neq$ const $)$. Если $\lambda(q)-$ не инвариант (1) при $u=\widetilde{v}_{0}$, то $\lambda(q)$ - множитель системы (1) лишь в случае $n=2$. Действительно, из (1), (5) оператор $D_{[}[\lambda][2,3]$, задающий множитель, имеет значение $D[\lambda]=\lambda+\lambda \underset{p}{\operatorname{div}} \widetilde{v}_{0}=(2-n) \lambda \mu^{*} \neq 0 \quad\left(\mu^{*} \neq 0, \mu=\ln |\lambda|\right)$.

Применение фазовой, пронзводящей функции канонизации $\lambda:=$ $=\lambda(q, P)$ ограничим для простоты двумя случаями - в первом условием подобия $L, K$ и $H, G$ соответственно по $q^{\prime}, q^{\prime}$ и $p, P$, во втором требованием преобразования подобия $(P=\lambda p)$. С учетом (3) в первом случае имеем

$$
\begin{gathered}
p=\psi(q, P)=\lambda^{-1}\left(P-\sigma \frac{\partial \mu_{0}}{\partial q^{\prime}}\right), \quad G=H(q, \psi), \\
K=\widetilde{L}\left(q, q^{\prime}\right)\left(\sigma \equiv q^{\prime} \cdot P=q \cdot p\right), \\
\lambda_{0}=\lambda(q, q /|q \cdot|), \\
\ln \left|\lambda_{0}\right|=\mu_{0}\left(q, q^{\prime}\right)=\mu_{0}\left(q, q^{*}\right)\left(q^{*} \cdot \nabla_{q^{*} \mu_{0}} \equiv 0, \frac{\partial^{2} K}{\partial q_{i}^{\prime} \partial q_{j}^{\prime}}=\lambda_{0}^{2} \frac{\partial^{2} L}{\partial q_{i} \partial q_{j}}\right) .
\end{gathered}
$$

Из (1), (2), (6) находим структуру и мощность канонизирующего регулятора

$$
\begin{gathered}
u=v_{1}(q, p)=\sigma E\left[\mu_{0}\right]-\lambda^{\prime} p-\sigma \frac{\partial \mu_{0}}{\partial q^{\prime}}, \\
W \equiv 0\left(E=\frac{\partial}{\partial q}-\frac{d}{d \tau} \frac{\partial}{\partial q^{\prime}}, f^{\prime} \equiv d f / d \tau\right) .
\end{gathered}
$$

Вид $v_{1}(q, p)$ получаем из $(3),(7)$ и равенств $q^{\prime}=\left(\lambda_{0}^{-1}\right) \partial H / \partial p, q^{*}=$ $=\partial H / \partial p$.

Рассмотрим теперь случай преобразования подобия $P=\lambda p$. Введем функцию $\tilde{H}(q, P) \equiv H\left(q, \lambda^{-1} P\right)$, где $\lambda$ принадлежит подклассу $\Lambda$ в $(2)$, заданному условиями

$$
\begin{gathered}
l[\tilde{H}](1-l[\mu])^{-1}=V(\mu), \quad 1-l[\mu] \neq 0, \\
\Delta_{P}(\tilde{H}+\mu V) \neq 0\left(l(f)=P \cdot \frac{\partial}{\partial P} f\right) .
\end{gathered}
$$


Первое из (8) эквивалентно $\left[{ }^{3}\right]$ существованию решения $G(q, P)$ системы $\nabla G=\nabla \tilde{H}+V(q, P) \nabla \mu$, равносильной $P=\lambda p(\Delta F=\partial F / \partial P)$. Остальные условия (8) обеспечивают обратимость $P \leftrightarrow p$ и $\Delta_{P} G \neq 0$. Отсюда из (3), (8) находим

$$
\begin{gathered}
G=G_{0}(q)+\int_{0}^{\mu} V(\xi) d \xi+\tilde{H}(q, P), \tilde{H}=\tilde{H}_{0}(q, e)+\int_{0}^{\mu} V(\xi) d \xi-\int_{0}^{\rho} V(\tilde{\mu}) d \varrho, \\
e=P /|P|, \varrho=\ln |P|, \tilde{\mu}=\tilde{\mu}(q, \varrho, e)=\mu(q, P)=\ln |\lambda|, \\
V=p \cdot q \cdot\left(G_{0}, \tilde{H}_{0} \in C_{1}(q, e)\right) .
\end{gathered}
$$

Здесь $G_{0}(q), \tilde{H}_{0}(q, e)$ - произвольные функции, $V(\mu)$ - задана для согласованных условиями (8) функций $\mu, H$, производящих $G$ и связь $P=\lambda p$. Из $^{(3)},(8),(9)$ имеем вид приводящего регулятора $v_{2}$ и его мощность

$$
v_{2}=-\nabla_{q} G_{0}-\mu^{\prime} p, W\left(v_{2}\right)=v_{2} \cdot \nabla_{p} H \neq 0\left(\mu^{\prime} \neq 0, \nabla_{q} G_{0}=0, f^{\prime}=d f / d \tau\right) .
$$

Здесь векторы $q, e-$ инварианты преобразования, $G(q, \lambda * p)-$ инвариант системы (1) при $u=v_{2}\left(\lambda(q, P) \equiv \lambda_{*}(q, p)\right)$. В подслучае натуральной системы

$$
H=1 / 2[p \cdot B(q) p] f H_{0}(q), B=B^{*}=\left\|b_{i j}\right\|>0 \quad(i, j=\overline{1, n})
$$

все множество допустимых для $(8)-(10)$ функций $\lambda, G$ имеет вид

$$
\begin{gathered}
\lambda^{-1}=f_{1} \exp F, G=1 / 4\left[f_{0}^{-1} f_{1}^{2}\left(\exp 2 F f f_{2}\right)\right], \\
F=f_{0}(P \cdot B P)\left(f_{0} \neq 0, f_{1} \neq 0, k=\overline{0,2}\right),
\end{gathered}
$$

где $f_{k}=f_{k}(q)$ - произвольные функции из класса $C_{1}(q) \quad(k=\overline{0,2})$.

Во всех рассмотренных случаях позиционная траектория $q[\tau]$ системы (2) сохраняется для (1), а импульс - преобразуется с учетом $(3): p[\tau]=\psi(q[\tau], P[\tau])$. Следовательно, вид решений (1) находим интеграцией $(2)$ в результате обращения функции

$$
t=\int_{0}^{\tau} \lambda^{-1}(q[\tau], P[\tau]) d \tau\left(\lambda=\lambda_{*}(q, p)=\lambda(q, \varphi)\right) .
$$

Легко показать, что при условии $\tau=\int_{0}^{t} \lambda * d t=$ fixconst вектор-функция $v_{k}(q, p)$ - стационарный регулятор системы (1) по функционалу

$$
I=\int_{0}^{t} \lambda_{*}(q, p)[(P \cdot \partial G / \partial P)-G] d t(\delta I=0, P=\varphi(q, p)) .
$$

\section{ЛИТЕРАТ У Р А}

1. Ч а лыгин С. А., Исследования по динамике неголономных систем, М.-Л., ГИТТЛ, 1949 , с. $28-38$.

2. Биркгоф Дж. Д., Динамические системы, М.-Л., ОГИЗ, ГИТТЛ, 1941, c. $34-54$.

3. Г у р а Э., Курс математического анализа, т. 2, М.-Л., ОНТИ, 1936, с. 510-532. 